Acta Crystallographica Section E

Structure Reports

Online

ISSN 1600-5368

\section{N-Benzyl-2-(2,6-dichlorophenoxy)- acetamide}

\section{Zhu-Bo Li, ${ }^{\text {a* }}$ Yong-Huang Luo, ${ }^{a}$ Wen-Liang Dong, ${ }^{b}$ Jing Li and Hua Zuo ${ }^{\mathrm{a}}$}

${ }^{a}$ College of Pharmaceutical Sciences, Southwest University, Chongqing 400716 , People's Republic of China, and 'Shandong University of Traditional Chinese Medicine, Jinan 250355, People's Republic of China

Correspondence e-mail: lizhubo2007@163.com

Received 8 July 2008; accepted 18 July 2008

Key indicators: single-crystal X-ray study; $T=298 \mathrm{~K}$; mean $\sigma(\mathrm{C}-\mathrm{C})=0.003 \AA$; $R$ factor $=0.045 ; w R$ factor $=0.132 ;$ data-to-parameter ratio $=18.9$.

The structure determination of the title compound, $\mathrm{C}_{15} \mathrm{H}_{13} \mathrm{Cl}_{2} \mathrm{NO}_{2}$, was undertaken as part of a project on the interaction of small molecules with proteins. In the crystal structure, the dihedral angle between the two aryl rings is $40.71(11)^{\circ}$. The molecules are connected via $\mathrm{N}-\mathrm{H} \cdots \mathrm{O}$ hydrogen bonding into chains, which extend in the direction of the $b$ axis.<smiles>O=C(COc1c(Cl)cccc1Cl)NCc1ccccc1</smiles>

\section{Experimental}

\section{Crystal data}

$\mathrm{C}_{15} \mathrm{H}_{13} \mathrm{Cl}_{2} \mathrm{NO}_{2}$

$M_{r}=310.16$

Orthorhombic, $\mathrm{Pbca}$

$a=14.8886(10) \AA$ $b=8.6579(6) \AA$

$V=2963.1$

$Z=8$
Mo $K \alpha$ radiation
$\mu=0.44 \mathrm{~mm}^{-1}$

$T=298(2) \mathrm{K}$

$0.20 \times 0.20 \times 0.10 \mathrm{~mm}$

Data collection

Bruker APEXII CCD area-detector diffractometer

Absorption correction: multi-scan (SADABS; Bruker, 2005)

$T_{\min }=0.918, T_{\max }=0.958$

Refinement

$R\left[F^{2}>2 \sigma\left(F^{2}\right)\right]=0.044$

$w R\left(F^{2}\right)=0.131$

$S=1.02$

3412 reflections

16445 measured reflections 3412 independent reflections 2103 reflections with $I>2 \sigma(I)$

$R_{\text {int }}=0.040$

181 parameters

$\mathrm{H}$-atom parameters constrained

$\Delta \rho_{\max }=0.20{\mathrm{e} \AA^{-3}}^{-3}$

$\Delta \rho_{\min }=-0.34$ e $\AA^{-3}$

Table 1

Hydrogen-bond geometry $\left(\AA,^{\circ}\right)$.

\begin{tabular}{lllll}
\hline$D-\mathrm{H} \cdots A$ & $D-\mathrm{H}$ & $\mathrm{H} \cdots A$ & $D \cdots A$ & $D-\mathrm{H} \cdots A$ \\
\hline $\mathrm{N} 1-\mathrm{H} 1 A \cdots \mathrm{O} 1$ & 0.86 & 2.23 & $2.644(2)$ & 109 \\
$\mathrm{~N} 1-\mathrm{H} 1 A \cdots \mathrm{O} 2^{\mathrm{i}}$ & 0.86 & 2.31 & $2.970(2)$ & 133 \\
\hline
\end{tabular}

Symmetry code: (i) $-x+\frac{3}{2}, y+\frac{1}{2}, z$.

Data collection: $A P E X 2$ (Bruker, 2005); cell refinement: $A P E X 2$; data reduction: $A P E X 2$; $\operatorname{program}(\mathrm{s})$ used to solve structure: $S I R 97$ (Altomare et al., 1999); program(s) used to refine structure: SHELXL97 (Sheldrick, 2008); molecular graphics: SHELXTL (Sheldrick, 2008); software used to prepare material for publication: Win $G X$ (Farrugia, 1999).

This study was supported by the Key Program Projects of the Municipal Natural Science Foundation of Chongqing, China (grant No. CSTC, 2007AC1042).

Supplementary data and figures for this paper are available from the IUCr electronic archives (Reference: NC2111).

\title{
References
}

Altomare, A., Burla, M. C., Camalli, M., Cascarano, G. L., Giacovazzo, C., Guagliardi, A., Moliterni, A. G. G., Polidori, G. \& Spagna, R. (1999). J. Appl. Cryst. 32, 115-119.

Bruker (2005). SADABS and APEX2. Bruker AXS Inc., Madison, Wisconsin, USA.

Farrugia, L. J. (1999). J. Appl. Cryst. 32, 837-838.

Sheldrick, G. M. (2008). Acta Cryst. A64, 112-122. 


\section{supporting information}

Acta Cryst. (2008). E64, o1610 [doi:10.1107/S1600536808022514]

\section{N-Benzyl-2-(2,6-dichlorophenoxy)acetamide}

\section{Zhu-Bo Li, Yong-Huang Luo, Wen-Liang Dong, Jing Li and Hua Zuo}

\section{S1. Experimental}

A solution of 2,6-dichlorophenol (1.0 mmol), $N$-benzyl-2-chloroacetamide (1.1 mmol), $\mathrm{K}_{2} \mathrm{CO}_{3}(1.1 \mathrm{mmol})$ in $\mathrm{CH}_{3} \mathrm{CN}(20$ $\mathrm{ml}$ ) was refluxed for $3 \mathrm{~h}$ and afterwards cooled down to room temperature. The solvent was removed under reduced pressure and the residue was poured into water and adjusted to $\mathrm{pH} 6-7$. with dilute hydrochloric acid (10\%) and extracted with ethyl acetate, washed with brine and dried over anhydrous $\mathrm{MgSO}_{4}$ to obtain the corresponding crude product. The product was obtained by column chromatography on silica gel using ethyl acetate as eluent. (yield 90\%). Crystals suitable for X-ray diffraction were obtained by slow cooling of a solution of the solid in ethyl acetate/hexane at room temperature for $4 \mathrm{~d}$.

\section{S2. Refinement}

All $\mathrm{H}$ atoms were placed in geometrically calculated positions and refined using a riding model with $\mathrm{C}-\mathrm{H}=0.97 \AA$ (for $\mathrm{CH}_{2}$ groups) and $0.96 \AA$ (for $\mathrm{CH}_{3}$ groups), their isotropic displacement parameters were set to 1.2 times (1.5 times for $\mathrm{CH}_{3}$ groups) the equivalent displacement parameter of their parent atoms.

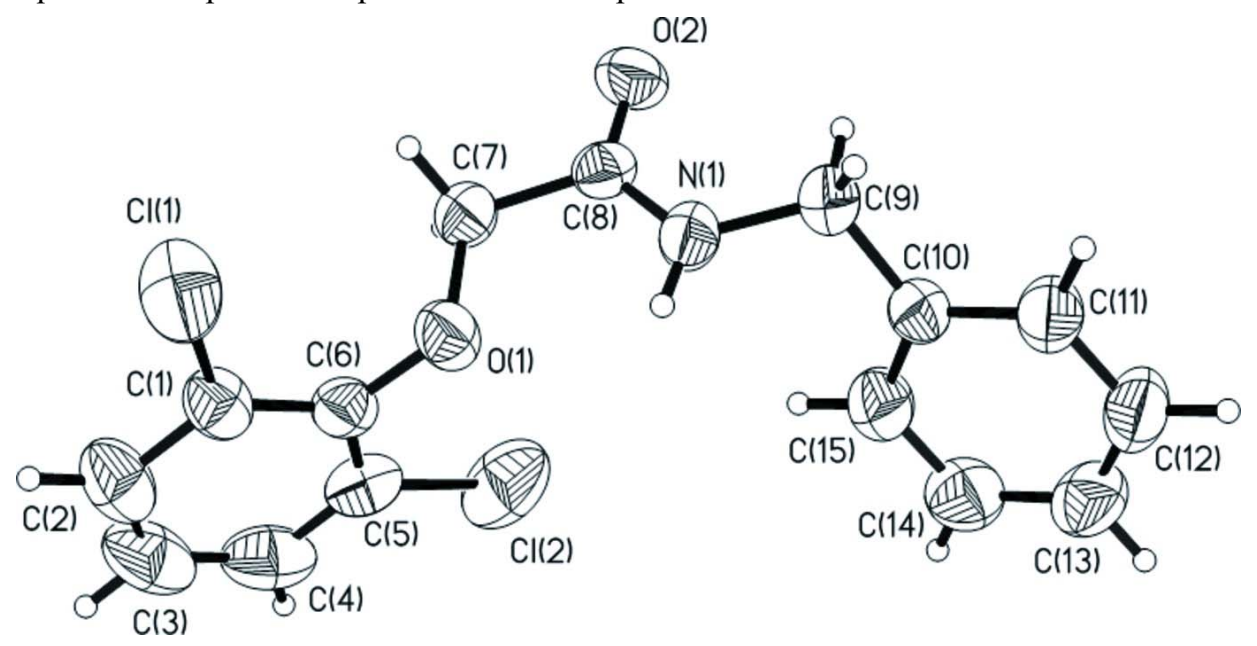

\section{Figure 1}

The molecular structure of the title compound with labelling and displacement ellipsoids drawn at the $50 \%$ probability level. 


\section{N-Benzyl-2-(2,6-dichlorophenoxy)acetamide}

Crystal data

$\mathrm{C}_{15} \mathrm{H}_{13} \mathrm{Cl}_{2} \mathrm{NO}_{2}$

$M_{r}=310.16$

Orthorhombic, $\mathrm{Pbca}$

$a=14.8886(10) \AA$

$b=8.6579(6) \AA$

$c=22.9867(14) \AA$

$V=2963.1(3) \AA^{3}$

$Z=8$

$F(000)=1280$

\section{Data collection}

Bruker SMART CCD area-detector diffractometer

Radiation source: fine-focus sealed tube Graphite monochromator

$\varphi$ and $\omega$ scans

Absorption correction: multi-scan

(SADABS; Bruker, 2005)

$T_{\min }=0.918, T_{\max }=0.958$

\section{Refinement}

Refinement on $F^{2}$

Least-squares matrix: full

$R\left[F^{2}>2 \sigma\left(F^{2}\right)\right]=0.045$

$w R\left(F^{2}\right)=0.131$

$S=1.02$

3412 reflections

181 parameters

0 restraints

Primary atom site location: structure-invariant direct methods
$D_{\mathrm{x}}=1.391 \mathrm{Mg} \mathrm{m}^{-3}$

Mo $K \alpha$ radiation, $\lambda=0.71073 \AA$

Cell parameters from 2733 reflections

$\theta=2.2-21.8^{\circ}$

$\mu=0.44 \mathrm{~mm}^{-1}$

$T=298 \mathrm{~K}$

Block, colorless

$0.20 \times 0.20 \times 0.10 \mathrm{~mm}$

16445 measured reflections

3412 independent reflections

2103 reflections with $I>2 \sigma(I)$

$R_{\text {int }}=0.040$

$\theta_{\text {max }}=27.5^{\circ}, \theta_{\min }=1.8^{\circ}$

$h=-19 \rightarrow 18$

$k=-10 \rightarrow 11$

$l=-20 \rightarrow 29$

Secondary atom site location: difference Fourier map

Hydrogen site location: inferred from neighbouring sites

$\mathrm{H}$-atom parameters constrained

$w=1 /\left[\sigma^{2}\left(F_{\mathrm{o}}^{2}\right)+(0.0631 P)^{2}+0.3666 P\right]$

where $P=\left(F_{\mathrm{o}}{ }^{2}+2 F_{\mathrm{c}}{ }^{2}\right) / 3$

$(\Delta / \sigma)_{\max }=0.001$

$\Delta \rho_{\max }=0.20 \mathrm{e} \AA^{-3}$

$\Delta \rho_{\min }=-0.34$ e $\AA^{-3}$

\section{Special details}

Geometry. All e.s.d.'s (except the e.s.d. in the dihedral angle between two 1.s. planes) are estimated using the full covariance matrix. The cell e.s.d.'s are taken into account individually in the estimation of e.s.d.'s in distances, angles and torsion angles; correlations between e.s.d.'s in cell parameters are only used when they are defined by crystal symmetry. An approximate (isotropic) treatment of cell e.s.d.'s is used for estimating e.s.d.'s involving 1.s. planes.

Refinement. Refinement of $F^{2}$ against ALL reflections. The weighted $R$-factor $w R$ and goodness of fit $S$ are based on $F^{2}$, conventional $R$-factors $R$ are based on $F$, with $F$ set to zero for negative $F^{2}$. The threshold expression of $F^{2}>\sigma\left(F^{2}\right)$ is used only for calculating $R$-factors(gt) $e t c$. and is not relevant to the choice of reflections for refinement. $R$-factors based on $F^{2}$ are statistically about twice as large as those based on $F$, and $R$ - factors based on ALL data will be even larger.

Fractional atomic coordinates and isotropic or equivalent isotropic displacement parameters $\left(\AA^{2}\right)$

\begin{tabular}{lllll}
\hline & $x$ & $y$ & $z$ & $U_{\text {iso }} * / U_{\text {eq }}$ \\
\hline N1 & $0.74097(11)$ & $0.0194(2)$ & $0.64026(7)$ & $0.0521(4)$ \\
H1A & 0.7004 & 0.0734 & 0.6230 & $0.063 *$ \\
C11 & $0.74730(5)$ & $0.13003(7)$ & $0.41952(3)$ & $0.0787(2)$ \\
C12 & $0.55214(5)$ & $-0.24316(9)$ & $0.55609(4)$ & $0.0985(3)$ \\
O1 & $0.68835(9)$ & $-0.00499(15)$ & $0.53078(6)$ & $0.0526(4)$
\end{tabular}




$\begin{array}{lllll}\text { O2 } & 0.84330(10) & -0.17088(18) & 0.62980(6) & 0.0591(4) \\ \text { C1 } & 0.65927(14) & 0.0023(2) & 0.42821(9) & 0.0515(5) \\ \text { C2 } & 0.60907(17) & -0.0417(3) & 0.38036(11) & 0.0679(7) \\ \text { H2A } & 0.6217 & -0.0014 & 0.3438 & 0.081^{*} \\ \text { C3 } & 0.54023(18) & -0.1457(3) & 0.38759(14) & 0.0802(8) \\ \text { H3A } & 0.5057 & -0.1746 & 0.3557 & 0.096^{*} \\ \text { C4 } & 0.52178(17) & -0.2071(3) & 0.44101(13) & 0.0761(8) \\ \text { H4A } & 0.4755 & -0.2783 & 0.4454 & 0.091^{*} \\ \text { C5 } & 0.57263(15) & -0.1625(3) & 0.48853(11) & 0.0603(6) \\ \text { C6 } & 0.64111(13) & -0.0555(2) & 0.48288(9) & 0.0466(5) \\ \text { C7 } & 0.76294(14) & -0.1008(3) & 0.54620(9) & 0.0522(5) \\ \text { H7A } & 0.8148 & -0.0717 & 0.5231 & 0.063^{*} \\ \text { H7B } & 0.7487 & -0.2076 & 0.5375 & 0.063^{*} \\ \text { C8 } & 0.78542(13) & -0.0856(2) & 0.60971(9) & 0.0433(5) \\ \text { C9 } & 0.75781(14) & 0.0471(3) & 0.70161(9) & 0.0597(6) \\ \text { H9A } & 0.7848 & 0.1484 & 0.7060 & 0.072^{*} \\ \text { H9B } & 0.8007 & -0.0286 & 0.7155 & 0.072^{*} \\ \text { C10 } & 0.67475(13) & 0.0393(2) & 0.73885(8) & 0.0471(5) \\ \text { C11 } & 0.67143(16) & 0.1244(3) & 0.78942(9) & 0.0586(6) \\ \text { H11A } & 0.7190 & 0.1894 & 0.7988 & 0.070^{*} \\ \text { C12 } & 0.59859(17) & 0.1148(3) & 0.82645(10) & 0.0696(7) \\ \text { H12A } & 0.5979 & 0.1718 & 0.8607 & 0.084^{*} \\ \text { C13 } & 0.52742(17) & 0.0216(3) & 0.81283(11) & 0.0693(7) \\ \text { H13A } & 0.4780 & 0.0165 & 0.8375 & 0.083^{*} \\ \text { C14 } & 0.52928(16) & -0.0638(3) & 0.76294(11) & 0.0697(7) \\ \text { H14A } & 0.4814 & -0.1283 & 0.7538 & 0.084^{*} \\ \text { C15 } & 0.60248(15) & -0.0545(3) & 0.72586(10) & 0.0609(6) \\ \text { H15A } & 0.6029 & -0.1123 & 0.6918 & 0.073^{*}\end{array}$

Atomic displacement parameters $\left(\AA^{2}\right)$

\begin{tabular}{lllllll}
\hline & $U^{11}$ & $U^{22}$ & $U^{33}$ & $U^{12}$ & $U^{13}$ & $U^{23}$ \\
\hline $\mathrm{N} 1$ & $0.0540(10)$ & $0.0620(11)$ & $0.0404(9)$ & $0.0117(8)$ & $0.0022(8)$ & $-0.0039(8)$ \\
C11 & $0.0982(5)$ & $0.0733(4)$ & $0.0644(4)$ & $-0.0303(4)$ & $-0.0038(3)$ & $0.0045(3)$ \\
C12 & $0.0904(5)$ & $0.1033(6)$ & $0.1019(6)$ & $-0.0081(4)$ & $0.0394(4)$ & $0.0184(4)$ \\
O1 & $0.0594(9)$ & $0.0521(8)$ & $0.0463(8)$ & $0.0140(7)$ & $-0.0084(7)$ & $-0.0094(6)$ \\
O2 & $0.0484(8)$ & $0.0657(9)$ & $0.0633(10)$ & $0.0121(7)$ & $-0.0112(7)$ & $-0.0085(7)$ \\
C1 & $0.0582(12)$ & $0.0460(12)$ & $0.0503(12)$ & $0.0008(10)$ & $-0.0087(10)$ & $-0.0080(9)$ \\
C2 & $0.0867(18)$ & $0.0606(14)$ & $0.0565(14)$ & $0.0067(13)$ & $-0.0203(12)$ & $-0.0093(11)$ \\
C3 & $0.0734(18)$ & $0.0740(18)$ & $0.093(2)$ & $0.0044(14)$ & $-0.0355(16)$ & $-0.0234(15)$ \\
C4 & $0.0485(13)$ & $0.0675(17)$ & $0.112(2)$ & $-0.0034(12)$ & $-0.0089(14)$ & $-0.0132(15)$ \\
C5 & $0.0466(12)$ & $0.0599(13)$ & $0.0745(16)$ & $0.0051(11)$ & $0.0084(11)$ & $-0.0035(11)$ \\
C6 & $0.0443(11)$ & $0.0458(11)$ & $0.0497(12)$ & $0.0084(9)$ & $-0.0027(9)$ & $-0.0087(9)$ \\
C7 & $0.0507(12)$ & $0.0603(13)$ & $0.0456(12)$ & $0.0127(10)$ & $0.0006(9)$ & $-0.0098(9)$ \\
C8 & $0.0362(10)$ & $0.0448(11)$ & $0.0488(12)$ & $-0.0023(9)$ & $0.0028(9)$ & $-0.0019(9)$ \\
C9 & $0.0522(12)$ & $0.0828(17)$ & $0.0441(12)$ & $-0.0021(11)$ & $0.0004(10)$ & $-0.0138(11)$ \\
C10 & $0.0481(11)$ & $0.0523(12)$ & $0.0409(11)$ & $0.0042(9)$ & $-0.0021(9)$ & $0.0014(9)$ \\
C11 & $0.0599(13)$ & $0.0665(15)$ & $0.0495(12)$ & $-0.0068(11)$ & $0.0043(11)$ & $-0.0085(10)$
\end{tabular}


supporting information

\begin{tabular}{lllllll}
$\mathrm{C} 12$ & $0.0749(16)$ & $0.0836(17)$ & $0.0503(13)$ & $-0.0039(14)$ & $0.0138(12)$ & $-0.0113(12)$ \\
$\mathrm{C} 13$ & $0.0610(14)$ & $0.0832(18)$ & $0.0636(16)$ & $-0.0015(13)$ & $0.0164(12)$ & $0.0084(13)$ \\
$\mathrm{C} 14$ & $0.0580(14)$ & $0.0770(16)$ & $0.0740(17)$ & $-0.0139(13)$ & $0.0017(12)$ & $0.0038(13)$ \\
$\mathrm{C} 15$ & $0.0621(14)$ & $0.0654(14)$ & $0.0552(14)$ & $-0.0045(12)$ & $-0.0020(11)$ & $-0.0112(11)$ \\
\hline
\end{tabular}

Geometric parameters $\left(A,{ }^{\circ}\right)$

\begin{tabular}{|c|c|c|c|}
\hline $\mathrm{N} 1-\mathrm{C} 8$ & $1.326(2)$ & $\mathrm{C} 7-\mathrm{C} 8$ & $1.504(3)$ \\
\hline $\mathrm{N} 1-\mathrm{C} 9$ & $1.452(3)$ & $\mathrm{C} 7-\mathrm{H} 7 \mathrm{~A}$ & 0.9700 \\
\hline $\mathrm{N} 1-\mathrm{H} 1 \mathrm{~A}$ & 0.8600 & $\mathrm{C} 7-\mathrm{H} 7 \mathrm{~B}$ & 0.9700 \\
\hline $\mathrm{C} 11-\mathrm{C} 1$ & $1.726(2)$ & $\mathrm{C} 9-\mathrm{C} 10$ & $1.506(3)$ \\
\hline $\mathrm{Cl} 2-\mathrm{C} 5$ & $1.730(3)$ & C9-H9A & 0.9700 \\
\hline $\mathrm{O} 1-\mathrm{C} 6$ & $1.378(2)$ & C9-H9B & 0.9700 \\
\hline $\mathrm{O} 1-\mathrm{C} 7$ & $1.431(2)$ & $\mathrm{C} 10-\mathrm{C} 11$ & $1.378(3)$ \\
\hline $\mathrm{O} 2-\mathrm{C} 8$ & $1.225(2)$ & $\mathrm{C} 10-\mathrm{C} 15$ & $1.381(3)$ \\
\hline $\mathrm{C} 1-\mathrm{C} 6$ & $1.379(3)$ & $\mathrm{C} 11-\mathrm{C} 12$ & $1.381(3)$ \\
\hline $\mathrm{C} 1-\mathrm{C} 2$ & $1.383(3)$ & C11-H11A & 0.9300 \\
\hline $\mathrm{C} 2-\mathrm{C} 3$ & $1.374(4)$ & $\mathrm{C} 12-\mathrm{C} 13$ & $1.368(3)$ \\
\hline $\mathrm{C} 2-\mathrm{H} 2 \mathrm{~A}$ & 0.9300 & $\mathrm{C} 12-\mathrm{H} 12 \mathrm{~A}$ & 0.9300 \\
\hline $\mathrm{C} 3-\mathrm{C} 4$ & $1.366(4)$ & $\mathrm{C} 13-\mathrm{C} 14$ & $1.365(3)$ \\
\hline $\mathrm{C} 3-\mathrm{H} 3 \mathrm{~A}$ & 0.9300 & $\mathrm{C} 13-\mathrm{H} 13 \mathrm{~A}$ & 0.9300 \\
\hline $\mathrm{C} 4-\mathrm{C} 5$ & $1.384(3)$ & $\mathrm{C} 14-\mathrm{C} 15$ & $1.386(3)$ \\
\hline $\mathrm{C} 4-\mathrm{H} 4 \mathrm{~A}$ & 0.9300 & $\mathrm{C} 14-\mathrm{H} 14 \mathrm{~A}$ & 0.9300 \\
\hline $\mathrm{C} 5-\mathrm{C} 6$ & $1.384(3)$ & $\mathrm{C} 15-\mathrm{H} 15 \mathrm{~A}$ & 0.9300 \\
\hline $\mathrm{C} 8-\mathrm{N} 1-\mathrm{C} 9$ & $122.78(18)$ & $\mathrm{O} 2-\mathrm{C} 8-\mathrm{N} 1$ & $124.39(19)$ \\
\hline $\mathrm{C} 8-\mathrm{N} 1-\mathrm{H} 1 \mathrm{~A}$ & 118.6 & $\mathrm{O} 2-\mathrm{C} 8-\mathrm{C} 7$ & $118.03(18)$ \\
\hline $\mathrm{C} 9-\mathrm{N} 1-\mathrm{H} 1 \mathrm{~A}$ & 118.6 & $\mathrm{~N} 1-\mathrm{C} 8-\mathrm{C} 7$ & $117.58(17)$ \\
\hline $\mathrm{C} 6-\mathrm{O} 1-\mathrm{C} 7$ & $114.23(14)$ & $\mathrm{N} 1-\mathrm{C} 9-\mathrm{C} 10$ & $113.75(17)$ \\
\hline $\mathrm{C} 6-\mathrm{C} 1-\mathrm{C} 2$ & $121.2(2)$ & $\mathrm{N} 1-\mathrm{C} 9-\mathrm{H} 9 \mathrm{~A}$ & 108.8 \\
\hline $\mathrm{C} 6-\mathrm{C} 1-\mathrm{Cl1}$ & $119.12(15)$ & $\mathrm{C} 10-\mathrm{C} 9-\mathrm{H} 9 \mathrm{~A}$ & 108.8 \\
\hline $\mathrm{C} 2-\mathrm{C} 1-\mathrm{C} 11$ & $119.65(18)$ & $\mathrm{N} 1-\mathrm{C} 9-\mathrm{H} 9 \mathrm{~B}$ & 108.8 \\
\hline $\mathrm{C} 3-\mathrm{C} 2-\mathrm{C} 1$ & $119.2(2)$ & $\mathrm{C} 10-\mathrm{C} 9-\mathrm{H} 9 \mathrm{~B}$ & 108.8 \\
\hline $\mathrm{C} 3-\mathrm{C} 2-\mathrm{H} 2 \mathrm{~A}$ & 120.4 & $\mathrm{H} 9 \mathrm{~A}-\mathrm{C} 9-\mathrm{H} 9 \mathrm{~B}$ & 107.7 \\
\hline $\mathrm{C} 1-\mathrm{C} 2-\mathrm{H} 2 \mathrm{~A}$ & 120.4 & $\mathrm{C} 11-\mathrm{C} 10-\mathrm{C} 15$ & $118.0(2)$ \\
\hline $\mathrm{C} 4-\mathrm{C} 3-\mathrm{C} 2$ & $120.9(2)$ & $\mathrm{C} 11-\mathrm{C} 10-\mathrm{C} 9$ & $119.02(19)$ \\
\hline $\mathrm{C} 4-\mathrm{C} 3-\mathrm{H} 3 \mathrm{~A}$ & 119.5 & $\mathrm{C} 15-\mathrm{C} 10-\mathrm{C} 9$ & $122.96(19)$ \\
\hline $\mathrm{C} 2-\mathrm{C} 3-\mathrm{H} 3 \mathrm{~A}$ & 119.5 & $\mathrm{C} 10-\mathrm{C} 11-\mathrm{C} 12$ & $121.1(2)$ \\
\hline $\mathrm{C} 3-\mathrm{C} 4-\mathrm{C} 5$ & $119.4(2)$ & $\mathrm{C} 10-\mathrm{C} 11-\mathrm{H} 11 \mathrm{~A}$ & 119.5 \\
\hline $\mathrm{C} 3-\mathrm{C} 4-\mathrm{H} 4 \mathrm{~A}$ & 120.3 & $\mathrm{C} 12-\mathrm{C} 11-\mathrm{H} 11 \mathrm{~A}$ & 119.5 \\
\hline $\mathrm{C} 5-\mathrm{C} 4-\mathrm{H} 4 \mathrm{~A}$ & 120.3 & $\mathrm{C} 13-\mathrm{C} 12-\mathrm{C} 11$ & $120.2(2)$ \\
\hline $\mathrm{C} 6-\mathrm{C} 5-\mathrm{C} 4$ & $121.0(2)$ & $\mathrm{C} 13-\mathrm{C} 12-\mathrm{H} 12 \mathrm{~A}$ & 119.9 \\
\hline $\mathrm{C} 6-\mathrm{C} 5-\mathrm{Cl} 2$ & $118.98(18)$ & $\mathrm{C} 11-\mathrm{C} 12-\mathrm{H} 12 \mathrm{~A}$ & 119.9 \\
\hline $\mathrm{C} 4-\mathrm{C} 5-\mathrm{Cl} 2$ & $120.0(2)$ & $\mathrm{C} 14-\mathrm{C} 13-\mathrm{C} 12$ & $119.7(2)$ \\
\hline $\mathrm{O} 1-\mathrm{C} 6-\mathrm{C} 1$ & $120.84(19)$ & $\mathrm{C} 14-\mathrm{C} 13-\mathrm{H} 13 \mathrm{~A}$ & 120.1 \\
\hline $\mathrm{O} 1-\mathrm{C} 6-\mathrm{C} 5$ & $120.90(19)$ & $\mathrm{C} 12-\mathrm{C} 13-\mathrm{H} 13 \mathrm{~A}$ & 120.1 \\
\hline $\mathrm{C} 1-\mathrm{C} 6-\mathrm{C} 5$ & $118.23(19)$ & $\mathrm{C} 13-\mathrm{C} 14-\mathrm{C} 15$ & $120.1(2)$ \\
\hline $\mathrm{O} 1-\mathrm{C} 7-\mathrm{C} 8$ & $111.28(15)$ & $\mathrm{C} 13-\mathrm{C} 14-\mathrm{H} 14 \mathrm{~A}$ & 120.0 \\
\hline
\end{tabular}




$\begin{array}{llll}\mathrm{O} 1-\mathrm{C} 7-\mathrm{H} 7 \mathrm{~A} & 109.4 & \mathrm{C} 15-\mathrm{C} 14-\mathrm{H} 14 \mathrm{~A} & 120.0 \\ \mathrm{C} 8-\mathrm{C} 7-\mathrm{H} 7 \mathrm{~A} & 109.4 & \mathrm{C} 10-\mathrm{C} 15-\mathrm{C} 14 & 120.9(2) \\ \mathrm{O} 1-\mathrm{C} 7-\mathrm{H} 7 \mathrm{~B} & 109.4 & \mathrm{C} 10-\mathrm{C} 15-\mathrm{H} 15 \mathrm{~A} & 119.5 \\ \mathrm{C} 8-\mathrm{C} 7-\mathrm{H} 7 \mathrm{~B} & 109.4 & \mathrm{C} 14-\mathrm{C} 15-\mathrm{H} 15 \mathrm{~A} & 119.5 \\ \mathrm{H} 7 \mathrm{~A}-\mathrm{C} 7-\mathrm{H} 7 \mathrm{~B} & 108.0 & & \\ & & & -154.72(17) \\ \mathrm{C} 6-\mathrm{C} 1-\mathrm{C} 2-\mathrm{C} 3 & -0.4(3) & \mathrm{C} 6-\mathrm{O} 1-\mathrm{C} 7-\mathrm{C} 8 & 1.3(3) \\ \mathrm{C} 11-\mathrm{C} 1-\mathrm{C} 2-\mathrm{C} 3 & 178.76(18) & \mathrm{C} 9-\mathrm{N} 1-\mathrm{C} 8-\mathrm{O} 2 & -178.76(19) \\ \mathrm{C} 1-\mathrm{C} 2-\mathrm{C} 3-\mathrm{C} 4 & -0.9(4) & \mathrm{C} 9-\mathrm{N} 1-\mathrm{C} 8-\mathrm{C} 7 & 174.13(18) \\ \mathrm{C} 2-\mathrm{C} 3-\mathrm{C} 4-\mathrm{C} 5 & 0.7(4) & \mathrm{O} 1-\mathrm{C} 7-\mathrm{C} 8-\mathrm{O} 2 & -5.8(3) \\ \mathrm{C} 3-\mathrm{C} 4-\mathrm{C} 5-\mathrm{C} 6 & 0.7(4) & \mathrm{O} 1-\mathrm{C} 7-\mathrm{C} 8-\mathrm{N} 1 & -126.9(2) \\ \mathrm{C} 3-\mathrm{C} 4-\mathrm{C} 5-\mathrm{C} 12 & -178.2(2) & \mathrm{C} 8-\mathrm{N} 1-\mathrm{C} 9-\mathrm{C} 10 & -151.9(2) \\ \mathrm{C} 7-\mathrm{O} 1-\mathrm{C} 6-\mathrm{C} 1 & -95.4(2) & \mathrm{N} 1-\mathrm{C} 9-\mathrm{C} 10-\mathrm{C} 11 & 30.6(3) \\ \mathrm{C} 7-\mathrm{O} 1-\mathrm{C} 6-\mathrm{C} 5 & 86.3(2) & \mathrm{N} 1-\mathrm{C} 9-\mathrm{C} 10-\mathrm{C} 15 & -9(3) \\ \mathrm{C} 2-\mathrm{C} 1-\mathrm{C} 6-\mathrm{O} 1 & -176.57(18) & \mathrm{C} 15-\mathrm{C} 10-\mathrm{C} 11-\mathrm{C} 12 & -176.7(2) \\ \mathrm{C} 11-\mathrm{C} 1-\mathrm{C} 6-\mathrm{O} 1 & 4.3(3) & \mathrm{C} 9-\mathrm{C} 10-\mathrm{C} 11-\mathrm{C} 12 & 1.1(4) \\ \mathrm{C} 2-\mathrm{C} 1-\mathrm{C} 6-\mathrm{C} 5 & 1.7(3) & \mathrm{C} 10-\mathrm{C} 11-\mathrm{C} 12-\mathrm{C} 13 & -0.9(4) \\ \mathrm{C} 11-\mathrm{C} 1-\mathrm{C} 6-\mathrm{C} 5 & -177.41(15) & \mathrm{C} 11-\mathrm{C} 12-\mathrm{C} 13-\mathrm{C} 14 & -0.7(3) \\ \mathrm{C} 4-\mathrm{C} 5-\mathrm{C} 6-\mathrm{O} 1 & 176.40(19) & \mathrm{C} 12-\mathrm{C} 13-\mathrm{C} 14-\mathrm{C} 15 & 176.8(2) \\ \mathrm{C} 12-\mathrm{C} 5-\mathrm{C} 6-\mathrm{O} 1 & -4.6(3) & \mathrm{C} 11-\mathrm{C} 10-\mathrm{C} 15-\mathrm{C} 14 & 0.7(4) \\ \mathrm{C} 4-\mathrm{C} 5-\mathrm{C} 6-\mathrm{C} 1 & -1.9(3) & \mathrm{C} 9-\mathrm{C} 10-\mathrm{C} 15-\mathrm{C} 14 & \\ \mathrm{C} 12-\mathrm{C} 5-\mathrm{C} 6-\mathrm{C} 1 & 177.05(16) & \mathrm{C} 13-\mathrm{C} 14-\mathrm{C} 15-\mathrm{C} 10 & \\ \end{array}$

Hydrogen-bond geometry $\left(\AA,{ }^{\circ}\right)$

\begin{tabular}{lllll}
\hline$D-\mathrm{H} \cdots A$ & $D-\mathrm{H}$ & $\mathrm{H} \cdots A$ & $D \cdots A$ & $D-\mathrm{H} \cdots A$ \\
\hline $\mathrm{N} 1-\mathrm{H} 1 A \cdots \mathrm{O} 1$ & 0.86 & 2.23 & $2.644(2)$ & 109 \\
$\mathrm{~N} 1-\mathrm{H} 1 A \cdots \mathrm{O} 2^{\mathrm{i}}$ & 0.86 & 2.31 & $2.970(2)$ & 133 \\
\hline
\end{tabular}

Symmetry code: (i) $-x+3 / 2, y+1 / 2, z$. 